\title{
Compliance to National Infant and Young Child Feeding Recommendation and Associated Factor Among Mothers of Children 6-23 months-of-age in Gombora District, Southern Ethiopia: Community Based Cross Sectional Study
}

\author{
Aberham Nuramo Chaimiso ${ }^{1}$, Terefe Markos Lodebo ${ }^{2,}$, , Meseret Temrat Gebretsadik ${ }^{3}$, \\ Abinet Arega Sadore ${ }^{4}$ \\ ${ }^{1}$ Gombora Woreda Health Office, Hadiya Zone, Southern Ethiopia \\ ${ }^{2}$ Department of Epidemiology, Institute of Health, Jimma University, Jimma, Ethiopia \\ ${ }^{3}$ Department of Population and Family Health, Institute of Health, Jimma University, Jimma, Ethiopia \\ ${ }^{4}$ Department of Health Education and Behavioral Science, Institute of Health, Jimma University, Jimma, Ethiopia \\ Email address: \\ abrnura2015@gmail.com (A. N. Chaimiso), teremark997@gmail.com (T. M. Lodebo), mesetamrat@yahoo.com (M. T. Gebretsadik), \\ abinetarege@gmail.com (A. A. Sadore) \\ ${ }^{*}$ Corresponding author
}

\section{To cite this article:}

Aberham Nuramo Chaimiso, Terefe Markos Lodebo, Meseret Temrat, Abinet Arega Sadore. Compliance to National Infant and Young Child Feeding Recommendation and Associated Factor Among Mothers of Children 6-23 months-of-age in Gombora District, Southern Ethiopia: Community Based Cross Sectional Study. International Journal of Clinical and Experimental Medical Sciences.

Vol. 3, No. 1, 2017, pp. 5-13. doi: 10.11648/j.ijcems.20170301.12

Received: March 14, 2017; Accepted: March 25, 2017; Published: April 26, 2017

\begin{abstract}
Background: Nutrition in infancy and early childhood is a critical determinant of health and productivity of the individual throughout life. During this period, appropriate, safe, nutritionally adequate and frequent feeding is essential. Despite this recommendation complementary feeding is commonly inappropriately practiced. There is no information compliance to national IYCF. Objective: To assess compliance to national IYCF recommendation and associated factors among mothers of children 6-23 months of age in Gombora district, Southern Ethiopia, 2016. Methods and Materials: Community-based cross-sectional study design was employed in Gombora district from March 1 to March 30, 2016. The data were collected from 379 respondents selected by simple random sampling technique using pre-tested and semistructured interviewer administered questionnaire. Bivariate analysis and multivariable logistic regression were employed to identify factors associated with compliance to IYCF. Results: Of the total 379 study subjects, $13.5 \%(95 \% \mathrm{CI}=10,17.5)$ of the respondents were reported as they comply with national IYCF recommendation; the odds being compliant to national IYCF recommendation was 5.26 times higher than for those respondents (9-12) grades of educational status and primary education (1-8) (AOR=5.26; 95\% CI:2.318, 11.914), accordingly the odds being compliant was 3.88 times higher than for those mothers of children within the age group (18-23) months and mothers of children age (6-11) months (AOR=3.88; 95\% CI:1.641, 9.162), the odds of being compliant to national IYCF for antenatal care visits greater than four visit was 3.95 times higher than other types of visit antenatal visit (AOR=3.95; 95\% CI:1.840, 8.488) and similarly the odds of being compliant was 2.95 times as much for those respondent who had postnatal care visit than no post-natal care visit $(\mathrm{AOR}=2.95 ; 95 \% \mathrm{CI}: 1.318,6.349)$ and the odds being non-compliant to national IYCF recommendation was $81 \%$ times among those who were not knowledgeable on indicators IYCF than those knowledgeable (AOR=0.19; $95 \%$ CI:0.075, 0.465 ) and the odds being compliant 6.02 times as much for those counseled on IYCF than those with no counseling on IYCF (AOR=6.02; 95\% CI:2.786, 12.998). Conclusion and recommendations: This study revealed that compliance to national IYCF recommendation was low. Nutrition education to mothers at every contact opportunity was recommended and mothers who were completed only primary education need more attention. All mothers must be encouraged to make antenatal care follow up at least four times.
\end{abstract}


Keywords: Compliance, IYCF, Children, Ethiopia

\section{Introduction}

Worldwide, more than 9 million children under 5 years of age die each year. Malnutrition underlies a majority of these U5 deaths, $70 \%$ of which occur in the first year of life [1]. Complementary feeding practice is a significant determinant of the nutritional status of infants. It is during this period of transitioning from exclusive breastfeeding to family foods that the incidence of malnutrition rises sharply resulting in deficits that are hard to compensate for in later childhood and in life [2]. Infant and Young Child Feeding is a set of wellknown and common recommendations for appropriate feeding of newborn and children less than two years of age and is a critical component of care in childhood [3]. It denotes the transition of an infant from exclusive breastfeeding to a family diet characterized by timely introduction of solid and semisolid foods with increasing amount, meal frequency and dietary diversity as the child gets older, while maintaining frequent breastfeeding [4].

World Health Organization recommend indicators for appropriate feeding for children age (6-23 months). Indicators that measure appropriate complementary feeding including timely initiation of complementary food with correct dietary diversity and meal frequency [5]. Poor feeding practices are a serious obstacle to attaining and maintaining health of this age group; about 220000 child lives could be saved every year with promotion of optimal breastfeeding and appropriate complementary feeding [6]. Child malnutrition, morbidity and mortality are reduced when mothers comply with the IYCF recommendations [7].

Compliance to the recommended (IYCF) practice has been shown to be very low. Globally, only a third of breastfeed infants 6-23 months of age met the criteria of recommended WHO IYCF practices [8]. In developing countries only less than one-third of 6-23 monthsold children are fed in accordance with IYCF practices as per recommended [9]. In Ethiopia, 44\% of children are stunted, 9\% are wasted, and $25 \%$ are underweight which informed the occurrence of both acute and chronic under nutrition (10). In Ethiopia only 51\% of children age 6-9 months received complementary foods and; $4 \%$ received foods from at least four food groups, while $48 \%$ were fed the minimum number of times or more and only $4 \%$ of youngest children 6-23 months are fed in accordance with IYCF practices as per recommended [11]

There exists a significant gap in explaining why in spite of the adoption of high impact IYCF policies and guidelines in Ethiopia, there is still low compliance to the recommended IYCF practice and high prevalence of malnutrition

\section{Methods and Materials}

\subsection{Study Area and Period}

The study was conducted in Gombora district, from March
1 to March 30 by using Community based cross sectional, study design was used.

\subsection{Population}

\subsubsection{Source Population}

All mothers children of age 6-23 months who reside in Gombora district.

\subsubsection{Study Population}

All mothers children 6-23 months of age living in randomly selected kebeles.

\subsubsection{Study Participants}

Mothers whose children 6-23 months of age selected for the study.

\subsection{Inclusion Criteria}

Mothers having children 6-23 months of age who reside in Gombora district.

\subsection{Exclusion Criteria}

Mothers children 6-23 months of age who reside in Gombora district and who Were very sick and unable to respond.

\subsection{Sample Size Determination and Sampling Technique}

\subsubsection{Sample Size Determination}

To determine the number of children age 6-23 months to be included in the study the single population proportion formula were used with the following assumptions: Since there were no previous studies which estimate mothers compliance with national IYCF recommendation in the area, a prevalence level that estimate maximum sample size (50\%) were considered, marginal error $(0.05)$, non-response rate of $10 \%$ or possible absenteeism and refusal to participate in the study, with $95 \%$ confidence level and alpha (0.05). Based on these assumptions the total samples size calculated using the formula indicated below gives 384 respondents.

$$
\mathrm{n}=x=\frac{\left(Z \frac{\alpha}{2}\right)^{2} P(1-P)}{d 2}
$$

The sample size is determined using single population proportion formula with the degrees of accuracy set at 0.05 .

$$
\mathrm{n}=\mathrm{Z}(\alpha / 2)^{2} \mathrm{pq} / d^{2}
$$

Where:

n- Sample size

$\mathrm{Z}-\mathrm{z}$ value for the chosen confidence interval (usually 0.95 $\alpha=0.05=1.96$ )

$\mathrm{P}=0.5$, since there were no previous studies the status of compliance with national IYCF recommendation; P taken as $50 \%$, Non response rate $=10 \%$ 


$$
\begin{gathered}
\mathrm{n}=(1.96)^{2 *}\left(0.5(1-0.5) /(0.05)^{2}+10 \%\right. \text { non-response rate } \\
\mathrm{n}=3.8416 * 0.25 / 0.0025=384
\end{gathered}
$$

Since the number of estimated mothers having children age 6-23 months in the district i.e. source population $(\mathrm{N}=$ 3817 ) is less than 10,000 , correction is needed as follows

$$
\mathrm{nf}=\frac{n}{1+\frac{n}{N}}=\frac{384}{1+\frac{384}{3817}}=349
$$

When a $10 \%$ non-response rate is considered, the total sample size is 384 mothers having children age 6-23 months.

\subsubsection{Sampling Technique}

The district was 22 rural and one urban kebele from which nine kebeles were selected using a lottery method. Proportional allocations were employed to obtain the sample size of the selected kebeles. Prior to the actual data collection, the list of study subjects were identified by using health management information system (HMIS) folder in the health post. Then those household numbers were picked up based on the existing house number in the HMIS folder. Finally, the study participants were selected by using random numbers generated by a computer program. The name and address of mothers having children age 6-23 months were specified and location were identified in collaboration with the kebele health extension workers and health development army.

The identified mothers were interviewed in each kebele till the numbers of sampled populations were completed. In some conditions like mothers away from home, the interviewers were re-visited the household at least three times and if it is failed to get the respondent, it was excluded from the survey and noted as non- response.

The 9 kebeles included in the study were: $\mathrm{k} 1$ = adeana, $\mathrm{k} 2$ = habicho, $\mathrm{k} 3=$ sagie, $\mathrm{k} 4=$ misa, $\mathrm{k} 5=1$ stole, $\mathrm{k} 6=(\mathrm{m} /$ gana $)$, $\mathrm{k} 7=\mathrm{a}$ rare, $\mathrm{k} 8=$ wondo and $\mathrm{k} 9=$ bole $($ see figure 1$)$

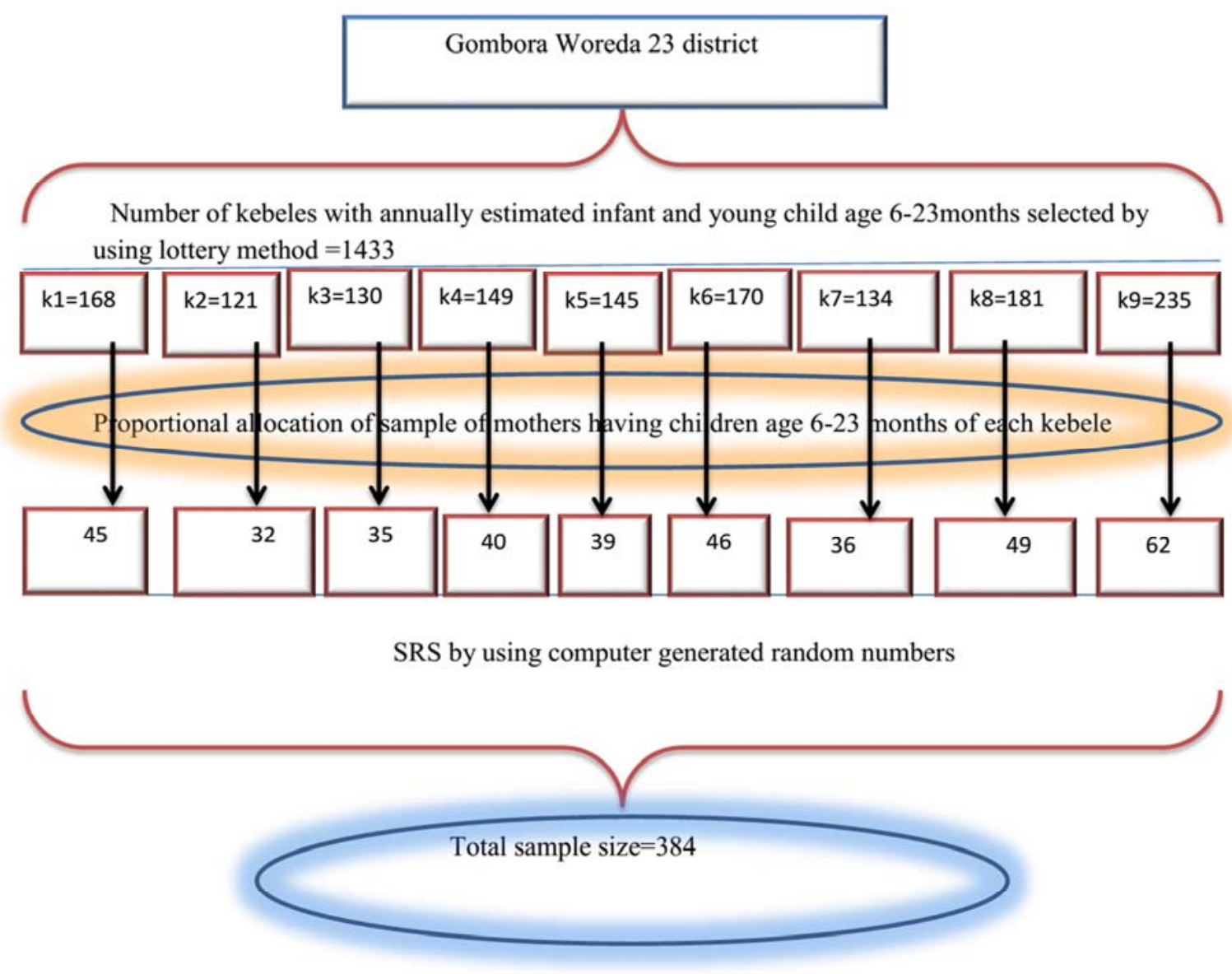

Figure 1. Schematic presentation of sampling procedure.

\subsection{Variables}

\subsubsection{Dependent Variable}

Compliance with national IYCF recommendation

\subsubsection{Independent Variables}

Socio-demographic related factor: - Age of mother, mother's ethnicity, mother's religion, marital status of the mother, mother's education status, household monthly income, residence

Child characteristics: - Age of child, sex of child, birth order of the child and desirability of pregnancy of index child

Maternal health service utilization related Factor: - 
antenatal care visit during pregnancy of index child, place of delivery (index child), and postnatal care visit (index child).

Facility related factors: - counseling on IYCF

Knowledge related factor: - Knowledge on indicators of IYCF and importance of feeding infant and young child.

Information related variable: - Exposure to media (radio, televisio)

\subsection{Data Collection Procedure}

The data were collected using semi structured pre-tested interviewer administered questionnaire adapted from different literatures and modified according to the local context by addressing important variables and EDHS 2011. The questionnaire was prepared in English and then translated to local language Hadiyisa and back translated to English to maintain consistency by two different individuals who know local language well. The content of the questionnaire include: maternal socio-demographic related variables, child characteristics related variable, maternal health service utilization related variable, information related variable, knowledge on indicators and importance of IYCF related variable and facility related variable and outcome variable were assessed based on the administration of interview questionnaire adopted from the WHO questionnaire 24-hour recall food diversity [12].

\subsection{Operational and Standard Definitions}

Compliant with IYCF recommendation: For this study a mother considered as compliant with IYCF recommendation if they fulfilled criteria of appropriate complementary feeding in preceding 24 hour recall of the survey which is equivalent to timely introduction solid and semi-solid with correct meal frequancy (i.e. minimum 2 times for 6-8 months of child and 3 times for 9-23 months of child) and dietary diversity (minimum 4 food type from seven food group) in 24 hours as per recommended.

Non-Compliant with IYCF recommendation: Among the above expected indicators of appropriate complementary feeding if at least one indicators were not fulfilled during 24 hour dietary recallperiod.

Maternal knowledge on indicators of IYCF: in this study from total of eight dichotomized questions 1 for correct and 0 for incorrect answer about recommended indicators who answer median score above were taken as having knowledgeable.

Maternal knowledge on importance IYCF: Those respondents from total of five dichotomized questions, 1 for correct and 0 for incorrect answer about importance of IYCF who answer median score above were taken as having knowledgeable.

Household monthly income: Monthly income below 1205 ETB was considered as poor and above 1205 ETB was considered as rich based on World Bank International poverty line 2015 [13].

\subsection{Data Entry, Processing and Analysis}

Data were entered into Epi data version 3.1 and then exported to SPSS version 20 for further analysis. Cleaning was made using frequencies. Descriptive statistics were used to describe the study variables. Then, binary logistic regression was used to examine the relationship between the proposed independent factors and dependent variable. Independent variables with Pvalue $\leq 0.25$ with compliance in bivariate analysis were candidate variables and entered into multivariable logistic regression to control the possible effect of confounders among independent variables. The model fitness for variables was assessed by Hosmer-Lemeshow Goodness of fit test. Adjusted odds ratio and corresponding $95 \%$ confidence intervals will be used to quantify the degrees of association between independent variables and mothers compliance with national IYCF recommendation. Finally, results were presented in summary statics, text, graph and tables.

\section{Ethical Consideration}

Ethical clearance and approval for the study was obtained from the Ethical review board of Jimma University, College of Health Science. Permission was obtained from woreda health office. Then the woreda health office sent official letters to local authority of all kebeles. The research presents no more than minimal risk of harm to subjects. Verbal consent was obtained from the participant's mother after informing them all the purpose, benefit, risk, the confidentiality of the information and the voluntary nature of participation in the study. Participants found to have mal practices regarding their infant or young child feeding had been counseled after the completion of the interview

\section{Result}

From the total of 384 sampled mothers having children 623 months of age, 379 were interviewed with response rate of $98.6 \%$.

\subsection{Socio-Demographic Characteristics of Mothers}

The age range of mothers considered in the study was 15-49 years, which is a childbearing age range. The mean age was 27 years $(\mathrm{SD} \pm 5.28)$. Among all respondents majority were rural residents 350 (92.3\%). Concerning maternal education, $77(20.3 \%)$ had no formal education. Out of whom, 205 (54.4\%) and $97(25.6 \%)$ have attended primary school and secondary school and above respectively. Majority of mothers 'occupation was housewives 362 (95.5\%). About 356 (93.9\%) respondents were protestant The predominant ethnic groups were hadiya which constitutes the larger proportion 365 (96.3\%) and other 14 (3.7\%). Among the study subjects 376 $(99.2 \%)$ of the respondents were married. (Table 1). 
Table 1. Socio demographic characteristics of mothers having children age 6-23 months in Gombora district, June 2016.

\begin{tabular}{|c|c|c|c|}
\hline Socio-Demographic characteristics & Categories & Numbers & Percentage \\
\hline \multirow{2}{*}{ Place of residence } & Urban & 29 & 7.7 \\
\hline & Rural & 350 & 92.3 \\
\hline \multirow{3}{*}{ Mothers' age } & $15-19$ & 5 & 1.3 \\
\hline & $20-34$ & 314 & 82.2 \\
\hline & $35-49$ & 60 & 15.7 \\
\hline \multirow[b]{2}{*}{ Marital status } & Married & 376 & 99.2 \\
\hline & Widowed & 1 & 0.3 \\
\hline \multirow{3}{*}{ Religion } & Protestant & 356 & 93.9 \\
\hline & Orthodox & 10 & 2.7 \\
\hline & Catholic & 13 & 3.4 \\
\hline \multirow{3}{*}{ Educational status } & No formal education & 76 & 20 \\
\hline & primary $(1-8)$ & 216 & 57 \\
\hline & Secondary (9-12) and above & 87 & 23 \\
\hline Occupation & house wife & 362 & 95.5 \\
\hline \multirow{4}{*}{ Ethnicity } & Hadiya & 365 & 96.3 \\
\hline & Kembata & 9 & 2.4 \\
\hline & alaba & 2 & 0.5 \\
\hline & silte & 3 & 0.8 \\
\hline
\end{tabular}

\subsection{Child, Household and Health Care Level Characteristics}

From sampled infant and young child about 207 (56.5\%) were female. The mean age were14 months (SD \pm 4.641 . Majority $222(58.6 \%)$. of the sample the birth order were third and above and $271(71.5 \%)$ desirability of pregnancy were planned. Regarding antenatal follow up about $149(39.3 \%)$ respondents were followed four or more time and 140 (36.9\%) received postnatal care visit. 339 (89.4\%) of the mothers were gave birth the youngest child in health facilities. (Table 2)

Table 2. Child, household, health care level characteristics and source of information of mothers having children aged 6-23 months, Gombora district, June 2016.

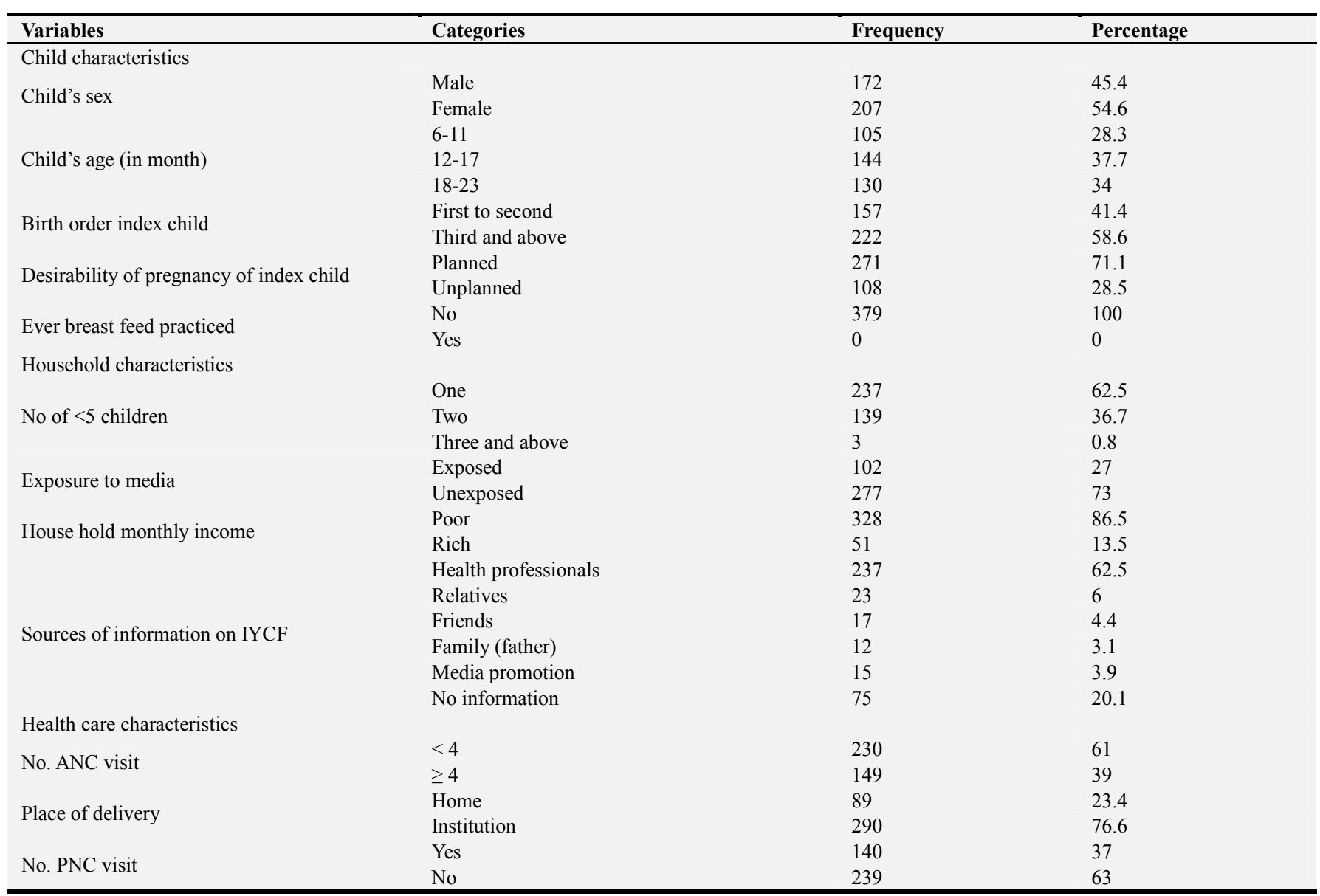


10 Aberham Nuramo Chaimiso et al: Compliance to National Infant and Young Child Feeding Recommendation and Associated Factor Among Mothers of Children 6-23 months-of-age in Gombora District, Southern Ethiopia: Community Based Cross Sectional Study

\subsection{Facility Related Factors}

Majority of them $(71.5 \%)$ were not counseled on IYCF (see figure 2)

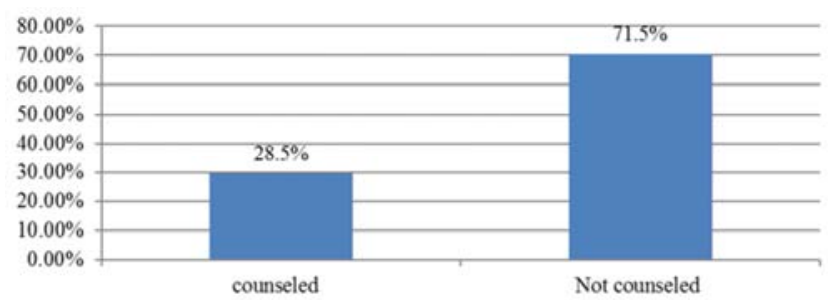

Figure 2. Diagrammatic presentation of mothers having children of 6-23 months age counseled to IYCF in Gombora district, South Ethiopia, June 2016.

\subsection{Maternal Knowledge on Importance of Feeding Infant and Young Child and Indicators IYCF}

Of the respondents, majority of them were knowledgeable (53.6\%) (see figure 3)

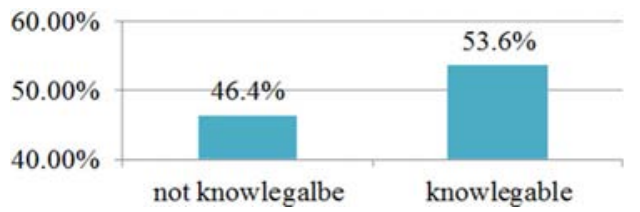

Figure 3. Mothers knowledge on importance IYCF in Gombora district, June 2016.

\subsection{Mother's Knowledge on Indicators IYCF}

Among study participants, most of them were knowledgeable on indicators (see figure 4)

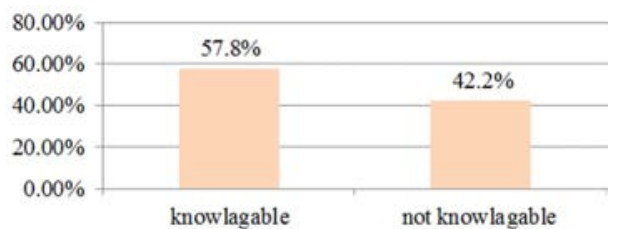

Figure 4. Mothers knowledge on indicators IYCF in Gombora district, June 2016.

\subsection{Complementary Feeding Practices}

Regarding feeding practices about 286 (75.5\%) mothers introduced complementary feeding as per recommended, 43 $(11.3 \%)$ mothers introduced early before 6 month, 45 $(11.9 \%)$ mothers initiated late after 6 months and $5(1.3 \%)$ mothers did not start complementary feeding at all. Accordingly 72 (19\%) mothers offered four or more food groups to their child and Simiraly $213(56.2 \%)$ practice below the recommended minimum meal frequancy. About $161(42.5 \%)$ mothers fed their children more than two times the day preceding 24 hour dietary recall period.

\subsection{Types of Food Practices}

Grain, roots and tubers were the most commonly taken food items by the children in 24 hours preceding the survey which constitute 362 (95.5\%), legumes and nuts 129 (34\%), dairy products $122(32.2 \%)$, vitamin-A rich foods 65 (17.2\%). (Table 3)

Table 2. Types of food groups consumed by the children age 6-23 months in Gombora district, June 2016.

\begin{tabular}{lll}
\hline Food groups & Frequency (n) & Percentage (\%) \\
\hline 1. Grains, roots and tubers & 362 & 95.5 \\
2. Legumes and nuts & 129 & 34 \\
3. Dairy products & 122 & 32.2 \\
4. Flesh food & 0 & 0 \\
5. Eggs & 68 & 17.9 \\
6. Vitamin A rich fruits and & 65 & 17.2 \\
Vegetables & 6 & 1.6 \\
7. Other fruits and vegetables & 6 & \\
\hline
\end{tabular}

\subsection{Compliance with National IYCF Recommendation}

The result of this study revealed that $13.5 \%$ ( $95 \% \mathrm{CI}=10$, 17.5) of the respondents compliant with IYCF recommendation in preceding 24 hour dietary recall period. About $86.5 \%$ mothers did not meet the national IYCF recommendation according to national IYCF guideline in 24 hour dietary recalls.

Reasons for non-compliance to IYCF recommendation (see figure 5)

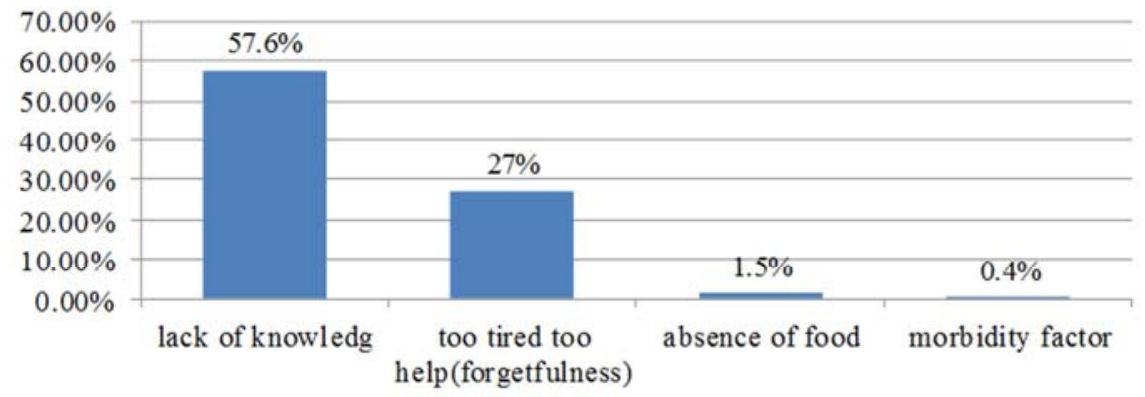

Figure 5. Reason for non-compliance to IYCF recommendation among mothers who had children of 6-23 months of age in Gombora district, June 2016.

\subsection{Factors Associated with Compliance to National IYCF Recommendation}

The multivariable analysis maternal educational level secondary (9-12) and above, mothers of child age (18-23) months, 
mothers knowledge on indicators IYCF, frequency of antenatal visits more than or equal to four, postnatal care visit and counseling on IYCF showed that significantly associated with compliance to IYCF recommendation.

Table 4. Factors independently associated with compliance to national IYCF recommendation in Gombora district, South Ethiopia, June 2016.

\begin{tabular}{|c|c|c|c|c|}
\hline \multirow[b]{2}{*}{ Variables } & \multicolumn{4}{|c|}{ Compliance with national IYCF recommendation } \\
\hline & Non-compliant & compliant & COR $(95 \% \mathrm{CI})$ & AOR $(95 \% C I)$ \\
\hline \multicolumn{5}{|l|}{ Age of mothers } \\
\hline$<25$ & $147(94.2 \%)$ & $9(5.8 \%)$ & $0.26(0.125,0.560)$ & $0.433(0.160,1.172)$ \\
\hline$\geq 25$ & $181(81.2 \%)$ & $42(18.8 \%)$ & 1 & 1 \\
\hline \multicolumn{5}{|l|}{ Educational status } \\
\hline No formal education & $71(93.4 \%)$ & $5(6.6 \%)$ & $0.78(0.277,2.164)$ & $0.58(0.174,1.920)$ \\
\hline Primary (grades 1-8) & $198(91.7 \%)$ & $18(8.3 \%)$ & 1 & 1 \\
\hline Secondary (9-12) and above & $59(67.8 \%)$ & $28(32.2 \%)$ & $5.22(2.669,10.097)$ & $5.26(2.318,11.914)^{*}$ \\
\hline \multicolumn{5}{|l|}{ Age of child (months) } \\
\hline 6-11 & $98(93.3 \%)$ & $7(6.7 \%)$ & 1 & 1 \\
\hline $12-17$ & $131(91 \%)$ & $13(9(\%)$ & $1.39(0.534,3.612)$ & $0.77(0.245,4.085)$ \\
\hline $18-23$ & $99(76.2 \%)$ & $31(23.8 \%)$ & $4.38(1.843,10.427)$ & $3.88(1.641,9.162)^{*}$ \\
\hline \multicolumn{5}{|l|}{ Pregnancy index child } \\
\hline Planned & $227(83.8 \%)$ & $44(16.5 \%)$ & 1 & 1 \\
\hline Unplanned & $101(93.5 \%)$ & $7(6.5 \%)$ & $0.36(0.156,0.821)$ & $0.44(0.159,1.240)$ \\
\hline \multicolumn{5}{|l|}{ No. ANC visit } \\
\hline$\geq 4$ & $116(77.9 \%)$ & $33(22.1 \%)$ & $3.35(1.807,6.212)$ & $3.95(1.840,8.488)^{*}$ \\
\hline$<4$ & $212(92.2 \%)$ & $18(7.8 \%)$ & 1 & 1 \\
\hline \multicolumn{5}{|l|}{ PNC visit } \\
\hline Yes & $111(79.3 \%)$ & $29(20.7 \%)$ & $2.58(1.415,4.693)$ & $2.97(1.389,6.349)^{*}$ \\
\hline No & $217(90.8 \%)$ & $22(9.2 \%)$ & 1 & 1 \\
\hline \multicolumn{5}{|l|}{$\begin{array}{l}\text { Knowledge on indicators of } \\
\text { IYCF }\end{array}$} \\
\hline Not knowledgeable & $152(95 \%)$ & $8(5 \%)$ & $0.13(0.098,0.472)$ & $0.19(0.075,0.465)^{*}$ \\
\hline Knowledgeable & $176(80.4 \%)$ & $43(19.6 \%)$ & 1 & 1 \\
\hline \multicolumn{5}{|l|}{ Counseled on IYCF } \\
\hline Yes & $76(70.4 \%)$ & $32(29.6 \%)$ & $5.58(2.995,10.411)$ & $6.02(2.786,12.998) *$ \\
\hline No & $252(93 \%)$ & $19(7 \%)$ & 1 & 1 \\
\hline
\end{tabular}

*Statistically significant at $p<0.05$ after being adjusted for other variables, $1=$ reference.

The multivariable analysis reviled that, the odds of being compliant to national IYCF was 5.26 times as much for those mothers educational level secondary (9-12) and above as compared to those mother who had primary education (1-8) (AOR $=5.26[95 \% \mathrm{CI}=2.318,11.914])$. Simiraly the odds of being compliant to national IYCF recommendation was 3.88 times as much for mothers of children within the age group (18-23) months as compared to mothers of infants in the age group (6-11) months $(\mathrm{AOR}=3.88[95 \% \mathrm{CI}=1.641,9.162])$. The odds being non-compliant to national IYCF recommendation was $81 \%$ times among those not knowledgeable on indicators of IYCF than those mothers having knowledgeable ( $\mathrm{AOR}=0.19[95 \% \mathrm{CI}=0.075,0.465])$. The odds of being compliant to national IYCF recommendation was 3.95 times as much for mother who had visited antenatal four times and above as compared to mothers who visited antenatal less than four times visit $(\mathrm{AOR}=3.95[95 \% \mathrm{CI}=1.840,8.488])$ and accordingly, the odds of being compliant to national IYCF recommendation was 2.97 times as much for mothers who had visited postnatal care visit as compared to mothers who had no postnatal care visit $(\mathrm{AOR}=2.97[95 \% \mathrm{CI}=1.318,6.349])$. The odds of being compliant to national IYCF recommendation was 6.02 times as much for mothers who had counseled on IYCF as compared to mothers who had no counseled on IYCF $(\mathrm{AOR}=6.02[95 \% \mathrm{CI}=2.786,12.998]$.

\section{Discussion}

Community based cross sectional studies determined compliance to national IYCF recommendation and associated factors among mothers having children in Gombora district of southern Ethiopia. The result of this study revealed that $13.5 \%(95 \% \mathrm{CI}=10,17.5)$ of the respondents compliant with IYCF recommendation and about $86.5 \%$ mothers did not meet the national IYCF recommendation for the appropriate complementary feeding according to IYCF guideline.

In this study one factor shown to be associated with compliance to national IYCF recommendation was mothers' educational status. The odds of being compliant to IYCF recommendation was 5.26 times as much for those motherseducational level secondary (9-12) and above compared to those mother who had primary education (1-8) $(\mathrm{AOR}=5.26(95 \% \mathrm{CI}=2.318,11.914))$.

This result was in agreement with the findings from Sirlanka, Nepal and Tanzania $[14,15,16]$

This could be because of educated mothers were more likely to have information and understand the education message.

Another variable shown to have a significant association with the compliance to national recommendation IYCF was found to be postnatal care visit. The odds of being compliant 
to national IYCF recommendation was 2.97 times as much for mothers who had visited postnatal care as compared to mothers who had no postnatal care visit $(\mathrm{AOR}=2.97$ $(95 \% \mathrm{CI}=1.318,6.349))$.

This result was in agreement with the findings in Tanzania that showed postnatal care visit was significantly associated with compliance to national IYCF recommendation [17].

This could be mothers received counseling from health workers during their postnatal visits.

Similarly, visiting antenatal four and more times was to have significant effects on mother's compliance with national IYCF recommendation.

The odds of being compliant to national IYCF recommendation was 3.95 times as much for mother who had visited antenatal four times and above as compared to mothers who visited antenatal less than four time visit $(\mathrm{AOR}=3.95(95 \% \mathrm{CI}=1.840,8.488))$

This finding was similar with northern Ghana [18].

The possible reason of this is that health care providers may educate mothers during their antenatal visits. Another variable which was associated with compliance to national IYCF recommendation were mothers of children within the age group (18-23) months. The odds of compliant to the IYCF recommendation was 3.88 times as much for mothers of children within the age group (18-23) months as compared to mothers of infants in the age group 6-11 months $(\mathrm{AOR}=3.88(95 \% \mathrm{CI}=1.641,9.162)$. This result was in similar with the findings in Nepal and Ghana, show that mothers of children within the age group (18-23) months significantly associated with compliance with national IYCF recommendation $[15,18]$. This could be some mothers couldn't introduce complementary feeding at six month.

Accordingly, another variable shown to have a significant association with compliance to IYCF recommendation was mother's knowledge on indicator IYCF. The odds being noncompliant was $81 \%$ times among those not knowledgeable on indicators IYCF than those knowledgeable on indicators IYCF $(\mathrm{AOR}=0.19(95 \% \mathrm{CI}=0.075,0.47))$. This finding was in agreement with study done in Udupi district of India and Bangladesh [19, 20]. The reason could be that knowledge helps mothers to have a good perception of prevention and treatment of malnutrition during infancy and early childhood.

In this study, other factor shown to have a significant association with the compliance with national IYCF recommendation was counseling on IYCF. The odds of complying to national IYCF recommendation was 6.02 times as much for mothers who had counseled on IYCF as compared to mothers who had no counseled on IYCF $(\mathrm{AOR}=6.02(95 \% \mathrm{CI}=2.786,12.998))$.

This finding was in agreement with the study done in Kenya [21].

Strength and limitations of study:

Even though, there is lack of adequate literatures in our country; this study had identified the level of compliance with comprising timing, dietary variety and meal frequency WHO IYCF recommendation for appropriate complementary feeding as a strength which can be information base line in the study area. The limitation of this study was data collected based on interviews and rely on the memory of mothers, there is possibility of recall bias, since it considers only $24 \mathrm{~h}$ (twenty four hour) feed, it may not accurately reflect their past feeding experience and socially desirable response. It does not take into account of the quality and amount of food provided.

\section{Conclusion and Recommendations}

\subsection{Conclusion}

Compliance to national IYCF recommendation was low in the area compared with other countries. About $86.5 \%$ mothers did not meet the recommendation for the appropriate complementary feeding according to national IYCF guideline. It was found that secondary level maternal education (9-12) and above, mothers of child ages (18-23) months, greater than or equal to four antenatal visits, postnatal care visit, and mothers' knowledge on indicators of IYCF and counseling on IYCF were factors that can increase compliance to IYCF.

\subsection{Recommendation}

Based on the study findings the following recommendations are forwarded

To woreda health office: -

Mothers who were completed only primary education need more attention.

Mothers having children of (6-11) month old should give special attention to designing nutrition education programs.

Lack of knowledge on indicators of IYCF is an important factor responsible for low compliance; strengthen monitoring and evaluation system regarding knowledge assessment.

Strengthen counseling services in health facilities at relevant maternal and child health contacts in primary health care services.

To health professionals at health facility:-

Maternal and child health service outlets should be used to transmit child feeding information it is important to educate mothers during every contact opportunity.

Promoting mothers to visit antenatal at least four times.

For researchers:-

Further research is recommended on compliance with the IYCF recommendation using actual feeding practices to overcomes the limitation of this study.

\section{Acknowledgements}

We would also like to extend thanks to Jimma University Institute of Health Sciences, Department of population and family health for providing me the opportunity to carry out this study.

Our appreciation goes to data collectors and supervisors for their active participation in the study

We would also like to extend our gratitude for Hadiya Zone health department, Gombora woreda health office and 
study participants for their valuable contribution in the realization of this study.

Above all, our gratitude and thanks goes to the Almighty God in all respects in our life.

\section{Declaration of Competing Interest}

Authors declare that no competing interests

\section{References}

[1] Infant and Young Child Feeding Practices. Collecting and Using Data: A Step-by- Step Guide. Cooperative for Assistance and Relief Everywhere, Inc. (CARE), 2010.

[2] Arabi M., Frongillo M., Avula R. and Mangasaryan N.: Infant and young child feeding in developing countries. Child development,2012; 83 (1), 32- 45.

[3] Federal Ministry of Health. Family Health Department Ethiopia: National strategy for infant and young child feeding. Addis Ababa: Federal Ministry of Health, Family Health Department Ethiopia; 2004.

[4] Victora C., Adair L., Fall C., Hallal P., Martorell R., Richter L. and Sachdev: Maternal and child under nutrition: consequences for adult health and human capital. The lancet, 2008. 371 (9609), 340--357.

[5] World Health Organization. Indicators for assessing infant and young child feeding practices: part 1: definitions: conclusions of a consensus meeting held 6-8 November 2007 in Washington DC, USA, 2008.

[6] Lozano R., Naghavi M., Foreman K., Lim S., Shibuya K., Aboyans V., Abraham J., Adair T., Aggarwal R. and Ahn S. Y: Global and regional mortality from 235 causes of death for 20 age groups in 1990 and 2010: 2013 The Lancet, 380 (9859), pp. 2095--2128. Martorell.

[7] Nduati, R: Supporting women to practice safe infant feeding: Kenya a case Study of the African experience. Paper presented at breast conferences. December 8 Th 2012, Wellstart.

[8] World Health Organization, The WHO Global Data Bank on Infant and Young Child Feeding. Geneva: 2013.

[9] Chessa K., Bernadette M. E., and Daelmans G. and Mercedesde O.: Poor Feeding Practices and Low Coverage of Key Nutrition Interventions: Pediatrics2011128; e1418. http://pediatrics.aappblications.org/content/128/6/e1418. full. html.

[10] Central Stastical Agency. Ethiopia Demographic and Health Survey 2011. Addis Ababa, Ethiopia, 2013.

[11] Ethiopia Demographic and Health Survey, Central Statistical Agency, Addis Ababa, Ethiopia ICF International, 2012.

[12] WHO, UNICEF, USAID, FANTA, AED, UC DAVIS, IFPRI: Indicators for assessing Infant and young child feeding practices part 2: measurement. Geneva: The World Health Organization; 2010

[13] World Bank International poverty line, 2015.

[14] Senarath U., Godakange S., Jayawickrama H., Siriwardena I. and Dibley M.: Determinants of inappropriate complementary feeding practices in young children in Sri Lanka Maternal \& child nutrition:2012;8 (1), 60-77

[15] Nira J., Kingsley E., Michael J. and Dibley A.: Compliance torecommended complementary feeding practices among mothers in young children in Nepal. Maternal and Child Nutrition: 2012; 8 (1), 45-59.

[16] Disha AD., Rawat R. and Subandoro N: Infant and young child feeding (IYCF) practice in Zambia and their association with child nutrition: African Scholarly Science Communication trust April 2012; 12(2):5899-5900.

[17] Rose V., Surinder K. and Baine K E: Factors associated with inappropriate complementary feeding practices among mothers children aged 6-23 months in Tanzania: Maternal Child Nutr.2012: Aug 23; 22925557.

[18] Mahama S., Asamoah L., Sofo M. and Irgard H-Z: Compliance to IYCF recommendation and factors associated among children 6-23 months in northern Ghana BMC Nutrition BMC trusted 2016 2:210.1186/s40795-015-0037-3.

[19] Meenakshi G; Compliance to infant and young child feeding practices and associated factor the same in Udupi district, Karnataka J Nut Res: 2015; 38-44ISSN: 2348-10.

[20] Kabir I., Dibley M. J. and Roy S. K: Determinants of inappropriate complementary feeding practices in infant and young children in Bangladesh Maternal \& Child Nutrition, 2012, 8 (s1), pp. 11--27.

[21] Mwitalina O: Compliance to recommended IYCF practice among mothers of infants and young children aged 6-23 months at mbagathi district hospital, Nairobi: 2012; H56/793. 